\title{
Bleeding time/Clotting time: Its Time to Change
}

\author{
Vikram Narang $^{1}$ (D) Keshav Sharma ${ }^{1} \cdot$ Neena Sood $^{1}$ \\ Received: 23 April 2020 / Accepted: 30 April 2020 / Published online: 15 May 2020 \\ (C) Association of Surgeons of India 2020
}

\section{Dear Editor,}

Bleeding time (BT) and clotting (CT) time tests are done in a clinical setup for the detection of hemostatic disorders, and it is presumed that normal BT-CT excludes the hemostatic disorders. The presumption is usually a part of a regular screening of a patient and can be taken as a prerequisite for the surgery and invasive procedures [1]. We intended to highlight that the over-relying of clinicians on BT and $\mathrm{CT}$ alone can miss the bleeding tendencies in asymptomatic patients. To comprehend our claim, we analyzed random 170 samples (in patients and out patients) with normal BT and CT for PT and APTT values.

Bleeding time was recorded by the modified Ivy's method, and clotting time was recorded by the capillary method. PT and APTT were analyzed in the ACL Top300 automated analyzer from the venous sample collected in a sodium citrate anticoagulant vacutainer. Of 170 patients, 55 (32.3\%) had prolonged PT, $20(11.7 \%)$ had prolonged APTT, and 17 $(10.0 \%)$ patients had both PT and APTT values above the upper limit.

Standardization of the diagnostic procedures is very important along with the technical competence while performing the $\mathrm{BT}$ and CT as the quality assurance and accuracy rely on this. Minor deviation like change in the size of sphygmomanometer cuff, depth of the incision made, lancet used, and the standard operating procedures followed can alter the results $[1,2]$. The modified methods commonly used add liitle valuable information for the patient care be it for diagnosing or monitoring a hemostatic disorder and hemostatic workup before surgery. BT-CT alone is not sensitive screening tests to detect

Vikram Narang

drvikramnarang@yahoo.com

Neena Sood

drneena_sood@yahoo.co.in

1 Department of Pathology, Dayanand Medical College and Hospital, Ludhiana, Punjab, India the hemostatic disorders. Their role in diagnosing a hemostatic disorder, or to rule out the bleeding tendency in a preoperative workup, is ambiguous. Despite thousands of these tests performed, not a single case of asymptomatic hemostatic disorder was detected. More often than not, these tests are carried out as an empirical legacy, perhabps to fulfill the predefined documentation requirements $[2,3]$.

Our experience and review of literature are in accordance to BT-CT tests which are obsolete and do not fulfill the criteria required for screening tests. Asymptomatic cases have no use of these tests, and the cases with clinical presentation are readily examined, and a more specific line of diagnostic procedures is available and practiced. It is time for the physicians, surgeons, anaesthesiologists and pathologists to modify their approaches.

\section{Compliance with Ethical Standards}

Conflict of Interest The authors declare that they have no conflict of interest

\section{References}

1. Rohrer MI, Micheloti MC, Nahrwold DL (1988) A prospective evaluation of the efficacyof preoperative coagulation testing. Ann Surg 208:554-557

2. Turnbull IM, Buck C (1987) The value of preoperative screening investigations in otherwise healthy individuals. Arch Intern Med t47:1101-1105

3. Lind SE (1991) The bleeding time does not predict surgical bleeding. Blood 77:2547-2552

Publisher's Note Springer Nature remains neutral with regard to jurisdictional claims in published maps and institutional affiliations. 